Este documento ha sido publicado como un capítulo del siguiente libro:

"La enseñanza del Derecho en tiempos de crisis. Nuevos retos docentes del Derecho Procesal”.Dir. Pocó i Junoy, J./Pérez Daudi, V./ Navarro Villanueva, C./ Cerrato Guri, E. Editorial: BOSCH. Barcelona, 2021.

\title{
TÉCNICAS PARA EL DESARROLLO DEL ROLE PLAYING EN REMOTO EN DERECHO PROCESAL
}

\author{
Dra. Andrea Spada Jiménez \\ Profesora Sustituta Interina de Derecho Procesal de la Universidad de Málaga
}

Sumario:

La utilización de los juegos de rol como un método de aprendizaje en el Derecho Procesal genera una asimilación de conocimientos por parte del alumnado lo bastante amplia como para que sea uno de los métodos más utilizados. Ahora bien, desarrollar el Role Playing de forma satisfactoria resulta bastante dificultoso por diversos motivos, y si a ello le sumamos la necesidad de desarrollar este método a través de un sistema remoto, genera una dificultad agravada. Sin embargo, en aras de facilitar dicha tarea en el presente trabajo expondré diversas técnicas utilizadas para su desarrollo; por un lado, aplicando el método a lo largo de todo el curso; y por otro, para ser aplicado en sesiones específicas de Role Playing. Las cuales, incluyen desde la preparación de la tarea, las instrucciones para el alumnado y la propia simulación en sentido estricto.

\section{INTRODUCCIÓN: EL ROLE PLAYING COMO MÉTODO DIDÁCTICO NECESARIO PARA EL DERECHO PROCESAL.}

La presente es una contribución que versa sobre la aplicación del role playing como una propuesta de técnica didáctica aplicable en la enseñanza de la asignatura de Derecho 
Este documento ha sido publicado como un capítulo del siguiente libro:

"La enseñanza del Derecho en tiempos de crisis. Nuevos retos docentes del Derecho Procesal”.Dir. Pocó i Junoy, J./Pérez Daudi, V./ Navarro Villanueva, C./ Cerrato Guri, E. Editorial: BOSCH. Barcelona, 2021.

Procesal que parte de la premisa de llevar a cabo un aprendizaje motivador, didáctico, profundo e interesante ${ }^{1}$.

Con las tareas en las que se asigna un rol a un estudiante o en las que el estudiante debe interpretar un determinado papel y desarrollarlo con base en los conocimientos teóricos que hubiera adquirido, se favorece el aprendizaje en cualquier tipo de materia a impartir, sobre todo en la enseñanza del derecho procesal donde cobra especial relevancia por la propia configuración de esa rama del derecho, la cual se encuentra especialmente enfocada en el aprendizaje del conjunto de normas que conforman el proceso y que se pueden escenificar en la celebración de audiencias, juicios orales, vistas o sesiones poniendo en práctica todos los conocimientos de normas procesales, procedimentales y a su vez los formales y de comportamiento que forman por completo el aprendizaje de un buen jurista.

Con el rol playing se desarrollan las diversas competencias inherentes a los estilos de aprendizaje activo $^{2}$, concretamente las cuatro capacidades expuestas por Kolb: La capacidad de "experiencia concreta, observación reflexiva, conceptualización abstracta y experimentación activa", aunque es cierto que para el autor el estilo de aprendizaje se lleva a cabo particularmente por el alumno y el desarrollo de esas capacidades dependerán del estilo de aprendizaje de cada individuo ${ }^{3}$. También es cierto que con este tipo de técnica se promueve el desarrollo de las cuatro capacidades.

\footnotetext{
${ }^{1}$ VARGAS GÓMEZ-URRUTIA, M. y M. HERRERA MOLINA, P. ¿Qué podemos aprender sobre Edith STEIN sobre la investigación y la enseñanza del Derecho?, Diario La Ley, núm. 7931, 2019. Esas cualidades son las expuestas por Edith Stein sobre su profesor Adolf Reinach, las cuales desde su visión constituyen un aprendizaje completo y motivador del Derecho, el cual comparto.

${ }^{2}$ KOLB, D. The learning Style Inventory: Technical Manual, Ma.: McBer, Boston, 1976. Cit. On. SAEZ LÓPEZ, J.M. Estilos de aprendizaje y métodos de enseñanza, Editorial UNED, Madrid, 2018.

${ }^{3}$ SAEZ LÓPEZ, J.M. Estilos de aprendizaje y métodos de enseñanza, Editorial UNED, Madrid, 2018.
} 
Este documento ha sido publicado como un capítulo del siguiente libro:

"La enseñanza del Derecho en tiempos de crisis. Nuevos retos docentes del Derecho Procesal”.Dir. Pocó i Junoy, J./Pérez Daudi, V./ Navarro Villanueva, C./ Cerrato Guri, E. Editorial: BOSCH. Barcelona, 2021.

Asimismo, a lo planteado se debe añadir un factor determinante y es la motivación del alumno, que cuando se consigue conlleva que el mismo demuestre interés por la materia y adopte una postura de aprendizaje activo. Para favorecerlo es necesario que el docente transmita entusiasmo e interés en el aprendizaje de sus alumnos utilizando técnicas de aprendizaje participativas como los juegos de rol y no solamente expositivas.

Ahora bien, para el desarrollo del role playing resulta imprescindible que se lleve a cabo teiendo una base teórica inicial para lo que se pueden utilizar otras técnicas.

\section{CUESTIONES PROBLEMÁTICAS PARA EL DESARROLLO DEL ROLE} PLAYING.

Aplicar una técnica de aprendizaje como esta de forma satisfactoria requiere tiempo, esfuerzo y voluntad por parte de los estudiantes y el profesor. A ello cabe añadir diversas dificultades que observamos cuando se comienza a configurar la tarea que son las siguientes:

Cuando se desarrollan sesiones específicas como puede ser a alumnos de prácticum o de posgrado, lo primero a lo que se enfrenta el profesor es que no se conoce al grupo al que nos enfrentamos para la realización de la actividad, agravado cuando encima se trata de llevarla a cabo de forma remota, lo que conlleva a la ignorancia por parte del docente en cuanto a si el grupo en general es proclive al aprendizaje activo o pasivo, o más bien, esta desmotivado y no demuestren interés para el desarrollo de las sesiones.

A su vez, el grupo suele ser bastante numeroso lo que complica la sesión ya que no todos los alumnos tienen la oportunidad de desarrollar el papel de jurista (abogado, procurador, fiscal, juez) sino que algunos deben jugar otros papeles de testigos o peritos. 
Este documento ha sido publicado como un capítulo del siguiente libro:

"La enseñanza del Derecho en tiempos de crisis. Nuevos retos docentes del Derecho Procesal”.Dir. Pocó i Junoy, J./Pérez Daudi, V./ Navarro Villanueva, C./ Cerrato Guri, E. Editorial: BOSCH. Barcelona, 2021.

Asimismo, para sesiones prácticas individuales de grado o posgrado, nos enfrentamos a un reducido espacio de tiempo para su desarrollo, ya que corresponde impartir clases prácticas a cada una de las asignaturas en sesiones de 4 o 6 horas en total, lo que conlleva que debamos ajustar la sesión a ese determinado periodo de tiempo disminuyendo así la efectividad de una evaluación formativa en una misma sesión ${ }^{4}$.

En cuanto a la aplicación de la técnica del role playing como actividad complementaria la dificultad para su desarrollo se encuadra sobre todo en que el grupo es muy numeroso y en el tiempo necesario que se debe invertir en la adquisición de conocimientos teóricos y conceptuales básicos y necesarios como para poder obtener una sesión de juegos de rol satisfactoria y fructífera.

Las cuestiones expuestas pueden surgir tanto para el desarrollo de la actividad presencial, como para el desarrollo de la actividad de forma remota, en las que se agravan cuando queremos realizar actividades donde existe una interacción. Ahora bien, estas dificultades han sido tenidas en cuenta para configurar las sesiones de role playing que aquí se plantean y que han sido aplicadas en la asignatura de Derecho Procesal Civil y Penal durante el curso con el mismo grupo, y en sesiones en remoto concretas de la asignatura de Prácticum I y II.

\footnotetext{
${ }^{4}$ En cuanto a las ventajas de la evaluación formativa. HAMODI, C.; LOPEZ PASTOR, V.M. y LOPEZ PASTOR, A.T. Medios, técnicas e instrumentos de evaluación formativa y compartida del aprendizaje en educación superior. Perfiles educativos. 2015, vol.37, n.147, pp.146-161.
} 
Este documento ha sido publicado como un capítulo del siguiente libro:

"La enseñanza del Derecho en tiempos de crisis. Nuevos retos docentes del Derecho Procesal”.Dir. Pocó i Junoy, J./Pérez Daudi, V./ Navarro Villanueva, C./ Cerrato Guri, E. Editorial: BOSCH. Barcelona, 2021.

\section{EL ROLE PLAYING APLICADO DURANTE EL CURSO PRESENCIAL O EN} REMOTO.

El determinar que se desarrolla la técnica de role playing durante el curso, no significa que todas las sesiones se vayan a desenvolver realizando simulaciones de casos, ni tampoco la utilización del método de casos, sino que más bien, se trata de inmiscuir al alumnado en la asignatura desde el primer día hasta el final del curso otorgándole un papel que seguramente vayan a desenvolver en su práctica laboral. En definitiva nos ayuda a llevar a cabo un método de aprendizaje complementado a su vez por otras técnicas que sea profundo, eficiente y motivante para mantener el interés del alumno.

El role playing como una técnica aplicable a lo largo del curso y no en sesiones individuales de simulación se plantea partiendo desde el primer día de clase hasta el final del curso planteándole al alumno el desarrollo del papel ya sea de abogado o procurador y generando complicidad y competitividad en el grupo desarrollándose de la siguiente manera:

Para comenzar, resulta desde mi punto de vista, necesario que el alumno obtenga un esquema general ${ }^{5}$ de los conocimientos que va a adquirir para que sepa lo que va a aprender, como conceptualizarlo, y como sistematizar los conocimientos en aras de generarle la seguridad y el interés necesario para tener una participación activa de

\footnotetext{
${ }^{5}$ Se debe tener en cuenta que es necesario que se realice una explicación del esquema de la forma más clara posible para que sea comprendido. En relación con la necesidad de unas exposiciones claras y comprensibles: DONDI, A. / NIEVA FENOLL, J. Diálogos sobre la docencia en derecho procesal (Dialogues about Teaching Procedural Law), Justicia: Revista de Derecho Procesal. J.B. Bosch, núm. 2, 2017, 81-

116.
} 
Este documento ha sido publicado como un capítulo del siguiente libro:

"La enseñanza del Derecho en tiempos de crisis. Nuevos retos docentes del Derecho Procesal”.Dir. Pocó i Junoy, J./Pérez Daudi, V./ Navarro Villanueva, C./ Cerrato Guri, E. Editorial: BOSCH. Barcelona, 2021.

aprendizaje $^{6}$. Para ello, es recomendable entregar al alumno el esquema referido con las fases y conceptos claves de la asignatura desde el primer día y hacer una referencia constante al mismo para que ellos lo vayan completando con cada nuevo conocimiento teórico que obtienen.

La forma de aplicar el role plaiyng durante el curso sin necesidad de configurar sesiones individuales es a través del planteamiento desde la fase inicial del proceso de un caso real con un expediente judicial que ellos puedan ver, porque se genera que la teoría se asimile casi de forma espontánea básicamente por el propio interés que le genera al alumno observar que las cuestiones teóricas que aprende son realmente útiles para el ejercicio de la profesión.

Lo recomendable, es que ese caso real, ha de ser configurado de forma que en el mismo resulten diversas vicisitudes procesales que debe resolver el alumno. Debiendo responder, cual abogado fuera del caso a cada una de las cuestiones procesales que se presentan haciendo uso de la Ley en la propia clase y desarrollando el papel de abogado planteando desde la forma del escrito, como la escena, como la institución a la cual debe dirigirse y las contestaciones que le puede dar a su supuesto cliente. Para ello el resto de compañeros pueden también adquirir un papel o plantear el rol de abogado de forma distinta. La labor del profesor es señalar el problema, encausar al alumno en la solución y dejar que el grupo participe activamente permitiendo que hagan de moderadores y compartan pantalla enseñando de donde han obtenido formularios y exista una interacción entre todo el grupo por supuesto con la cámara y micrófono encendida. Con ello se genera una capacidad

\footnotetext{
${ }^{6}$ KARINA CICERO, N. (2018) El primer día de clases. Revista de Derecho y Educación. Education and Law review, núm. 18. Defiende la necesidad de crear un vinculo entre los estudiantes y el profesor para promocionar esa participación activa.
} 
Este documento ha sido publicado como un capítulo del siguiente libro:

"La enseñanza del Derecho en tiempos de crisis. Nuevos retos docentes del Derecho Procesal”.Dir. Pocó i Junoy, J./Pérez Daudi, V./ Navarro Villanueva, C./ Cerrato Guri, E. Editorial: BOSCH. Barcelona, 2021.

reflexiva, activa, motivación y entretenimiento que en la presente era de la información y la tecnología parece tan necesario, y que conducen a un aprendizaje completo e interesante.

\section{SESIONES DE ROLE PLAYING.}

Para el desarrollo de las sesiones de role playing que se preparan como una actividad concreta como actividad complementaria durante el curso o como parte de la asignatura de práctica cuando se plantea de forma remota puede generar que los obstáculos expuestos se acrecienten. Sin embargo, mediante el presente plasmaré la preparación y el desarrollo de las sesiones tal y como fueron preparadas para la asignatura de Prácticum, las cuales se desarrollaron de forma muy satisfactoria y desde un punto de vista bastante innovador ante la dificultad de realizar juegos de rol en remoto.

Para la preparación de las sesiones se debe utilizar el Campus Virtual (plataforma Moodle) siendo la herramienta fundamental entre el profesor y el alumno sobre todo para la docencia no presencial ${ }^{7}$.

Preparar la sesión para el alumnado es relevante para asegurar el éxito de la actividad y para evitar dudas en los estudiantes, máxime cuando se trata de docencia no presencial.

Como se puede observar en la imagen del Campus virtual, para la actividad se utilizan diversas herramientas:

\footnotetext{
${ }^{7}$ Igualmente en una sociedad digital, el uso de las TIC por parte del profesorado es necesario y contribuyen a mejorar la calidad de la enseñanza. GARCIA CONTADOR, I., GUTIÉRREZ ESTEBAN, P. El rol docente en la sociedad digital. Digital Education Review - Number 38, 2020.
} 
Este documento ha sido publicado como un capítulo del siguiente libro:

"La enseñanza del Derecho en tiempos de crisis. Nuevos retos docentes del Derecho Procesal”.Dir. Pocó i Junoy, J./Pérez Daudi, V./ Navarro Villanueva, C./ Cerrato Guri, E. Editorial: BOSCH. Barcelona, 2021.

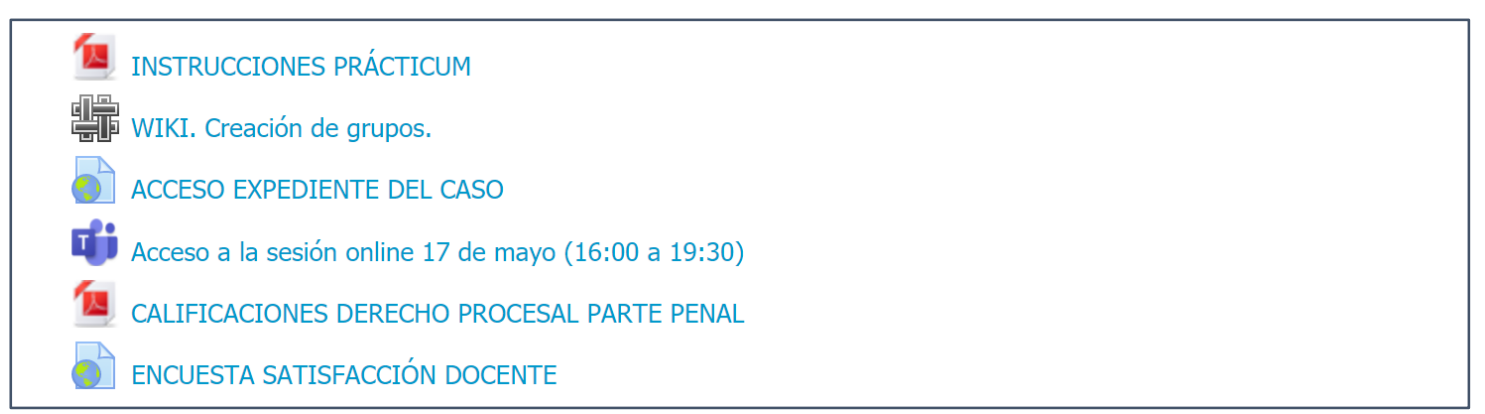

En cuanto al caso escogido, se trataba de un expediente judicial real sobre la comisión de un delito de estafa al que podían acceder a través del enlace expuesto y se iba a simular el juicio oral. Siendo defensora del aprendizaje colaborativo ${ }^{8}$ y para permitir que todos los alumnos participen en la tarea, se plantea mediante la herramienta wiki la creación de grupos en la cual cada alumno se asigna un rol de los disponibles y el grupo al que va a pertenecer. En el documento sobre las instrucciones de la tarea, especificaba como ha de prepararse la tarea por el alumno, como se desarrollará la sesión y en qué plataforma.

Para la preparación de la tarea, se indicaba que el alumno debía leer el expediente e informarse con las herramientas que considere sobre el tema y el desempeño de su papel en juicio.

En cuanto al desarrollo de las sesiones, lo ideal para el role playing sería poder contar con un programa como el de Second $\mathrm{Life}^{9}$, en los cuales se crean personajes y salas, se trata de una realidad virtual. De lo contrario, se recomienda utilizar una plataforma con la que se puedan crear salas por grupos como puede ser Microsoft Teams (MT). Una vez comenzada la sesión cada alumno compartió a través de la pantalla la información

\footnotetext{
${ }^{8}$ En cuanto a las ventajas del aprendizaje colaborativo: BARKLEY, E. F; PATRICIA CROSS; K. HOWELL MAJOR, C. Técnicas de aprendizaje colaborativo: Manual para el profesorado universitario, editorial Morata, España, 2007.

${ }^{9}$ MONTERROSO CASADO, E. / ESCUTIA ROMERO, R. La enseñanza práctica en 3D: Juicio virtual, @ tic. revista d'innovació educativa, núm. 6, 2011, 46-54.
} 
Este documento ha sido publicado como un capítulo del siguiente libro:

"La enseñanza del Derecho en tiempos de crisis. Nuevos retos docentes del Derecho Procesal”.Dir. Pocó i Junoy, J./Pérez Daudi, V./ Navarro Villanueva, C./ Cerrato Guri, E. Editorial: BOSCH. Barcelona, 2021.

recabada para preparar el juicio oral, desde videos en Youtube, enciclopedias jurídicas, libros y leyes y entre todos analizamos, para proceder a una segunda fase en la que reunidos en las salas de grupos de MT se preparaban la sesión en un periodo de 30 minutos y finalmente se celebraba la sesión de juicio oral. Para las calificaciones se tenia en cuenta tanto el trabajo en grupo en cuanto a la simulación para que fuera equitativo por el limite de tiempo y asignación de roles y el trabajo individual para aquellos que compartieron el material obtenido.

\section{RESULTADOS Y CONCLUSIONES.}

Los resultados obtenidos de las sesiones de role playing de acuerdo con lo expresado por los alumnos fueron muy satisfactorios, entendieron a la perfección la tarea, tuvieron un papel participativo y activo durante toda la sesión, compartieron la documentación obtenida para el desarrollo de las sesiones y expresaron haber aprendido mucho por la preparación de la tarea colaborativa y cooperativa. Asimismo, demostraron ostentar los conocimientos teóricos, prácticos y de comportamiento para haber desarrollado la tarea de forma exitosa y con motivación.

Habiendo expuesto a lo largo del trabajo las diversas capacidades que se obtienen con el role playing, independientemente de si es presencial o no presencial, cabe disponer como conclusión general que a pesar de las dificultades que se presentan, es factible el desarrollo del role playing en remoto y hasta se obtiene la ventaja de poder fomentar un aprendizaje más colaborativo y cooperativo así como adecuado para la sociedad digital en la que el alumnado está inmerso obteniendo el interés y motivación necesario. La clave consiste en demostrarle al alumno que el tiempo invertido en clase es más productivo que 
Este documento ha sido publicado como un capítulo del siguiente libro:

"La enseñanza del Derecho en tiempos de crisis. Nuevos retos docentes del Derecho Procesal”.Dir. Pocó i Junoy, J./Pérez Daudi, V./ Navarro Villanueva, C./ Cerrato Guri, E. Editorial: BOSCH. Barcelona, 2021.

el invertido en buscar la información por si mismo en Internet y que su formación universitaria se adecúa a la práctica laboral.

\section{BIBLIOGRAFÍA}

BARKLEY, E. F; PATRICIA CROSS; K. HOWELL MAJOR, C. Técnicas de aprendizaje colaborativo: Manual para el profesorado universitario, editorial Morata, España, 2007.

DONDI, A. / NIEVA FENOLL, J. Diálogos sobre la docencia en derecho procesal (Dialogues about Teaching Procedural Law), Justicia: Revista de Derecho Procesal. J.B. Bosch, núm. 2, 2017, 81-116.

GARCIA CONTADOR, I., GUTIÉRREZ ESTEBAN, P. El rol docente en la sociedad digital. Digital Education Review - Number 38, 2020.

HAMODI, C.; LOPEZ PASTOR, V.M. y LOPEZ PASTOR, A.T. Medios, técnicas e instrumentos de evaluación formativa y compartida del aprendizaje en educación superior. Perfiles educativos. 2015, vol.37, n.147, pp.146-161.

KARINA CICERO, N. (2018) El primer día de clases. Revista de Derecho y Educación. Education and Law review, núm. 18. Defiende la necesidad de crear un vinculo entre los estudiantes y el profesor para promocionar esa participación activa.

MONTERROSO CASADO, E. / ESCUTIA ROMERO, R. La enseñanza práctica en 3D: Juiciovirtual, @ tic. revista d'innovació educativa, núm. 6, 2011, 46-54.

SAEZ LÓPEZ, J.M. Estilos de aprendizaje y métodos de enseñanza, Editorial UNED, Madrid, 2018. 
Este documento ha sido publicado como un capítulo del siguiente libro:

"La enseñanza del Derecho en tiempos de crisis. Nuevos retos docentes del Derecho Procesal”.Dir. Pocó i Junoy, J./Pérez Daudi, V./ Navarro Villanueva, C./ Cerrato Guri, E. Editorial: BOSCH. Barcelona, 2021.

VARGAS GÓMEZ-URRUTIA, M. y M. HERRERA MOLINA, P. ¿Qué podemos aprender sobre Edith STEIN sobre la investigación y la enseñanza del Derecho?, Diario La Ley, núm. 7931, 2019. 